\title{
CloudAnalyst : A Survey of Load Balancing Policies
}

\author{
Sandip Patel \\ U \& P U. Patel Department of \\ Computer Engineering, \\ CHARUSAT University \\ Changa, Gujarat, India
}

\author{
Ritesh Patel \\ U \& P U. Patel Department of \\ Computer Engineering, \\ CHARUSAT University \\ Changa, Gujarat, India
}

\author{
Hetal Patel \\ U \& P U. Patel Department of \\ Computer Engineering, \\ CHARUSAT University \\ Changa, Gujarat, India
}

\author{
Seema Vahora \\ U \& P U. Patel Department of Computer Engineering, \\ CHARUSAT University \\ Changa, Gujarat, India
}

\begin{abstract}
Cloud computing is one of the incredible technology which enable the new vision for IT industry. Nowadays, it has become a strong alternative for startup large as well as small scale organizations that only use the resources which actually required based on pay as per use. As Cloud Computing is growing continuously and clients from different parts of the world are demanding for the various services and better outcomes, the load balancing has become the challenge for the cloud provider. To accurately manage the available resources of the different cloud provider, resources have to be properly selected according to the properties of task. Many algorithms have been proposed to provide efficient mechanisms and assigning the client's requests to available cloud nodes and aim to enhance the overall performance of the cloud and provide more satisfaction to user and efficient services. Initially this paper gives an introduction to cloud computing and load balancing. A detailed survey on different load balancing policy in cloud analyst, their advantages and drawback with obtainable solution and learn how to add new policy or customize existing load balancing policy.
\end{abstract}

\section{Keywords}

Cloud Computing, Cloud Analyst, Virtual Machine, Datacenter, Load balancing policy.

\section{INTRODUCTION}

"Cloud computing refers to computing on the Internet, as opposed to computing on a desktop" [1]. Standardizing the definition of cloud computing provided by The National Institute of Standards and Technology (NIST) [12]. NIST definition of cloud computing Cloud computing is a model for enabling convenient, on-demand network access to a shared pool of configurable computing resources like servers, networks, storage, applications etc., that can be rapidly provisioned and released with minimal management effort or service provider interaction [12].

Cloud computing is not an innovative technology, but rather a new operations model that carries together a set of present technologies to run business in an innovative way [9]. Cloud computing take the advantage of these present technologies to accomplish the economic and the technological requirements of today's demand for IT [9]. There are several technologies like vitalization, utility computing, grid computing, which share certain aspect with cloud computing [9]. Nowadays, Up and Down time of any IT industry is unpredictable so its require some flexible platform in which customer or client can increase capacity or add capabilities based on their resources requirement (resources can be a storage, platform, software, power and bandwidth) that's why cloud computing come in to picture which contains any subscription-based services that, in real time over the internet, and extends existing IT's competences.

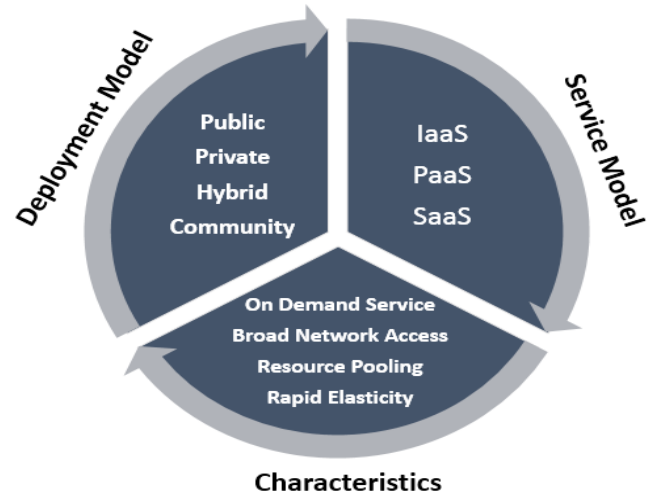

Fig 1: Cloud computing

As show in figure cloud computing is combination of three important factors characteristics, deployment model and service model.

In Section III, we cover Load balancing. In Section IV, we cover existing load balancing policy in cloud analyst with drawback and possible solution. In Section V, cloud analyst simulator discussed. In Section VI, simulated results of existing algorithms.is discussed. In VII implementation details discussed and finally the conclusion of survey is presented.

\section{LOAD BALANCING}

Load balancing of request distributes workloads across multiple computing resources, such as computers, computer cluster, disk drives, network links, CPU [15].

As technology growing faster, there are huge amount of user on internet so managing and fulfil their requirement, load balancer come in to picture which essentially ensure that they get spread workload equally to the all available server without any delay which help to accomplish a high user satisfaction, Maximum throughput with minimum response time [16]. Load balancer is not only design for cloud workload but also design for different purpose like DNS load balancer, database load balancer, website load balancer [15].Load balancing done on two level in cloud computing [10].

- VM level, mapping done between an applications which are uploaded on the cloud to virtual machine, the load balancer assigns the requested VM to physical computers which balance the load of numerous applications among PCs. 
- Host level, mapping done between virtual machine and host resources which help to proceeds multiple incoming requests of application.

\subsection{Classification of Load Balancing Algorithms}

In general, load balancing algorithms classified in to two key approaches based on that decisions making process: Static and Dynamic load balancing algorithms.

Static Load Balancing Algorithms: - Static algorithms are much simpler as compared to dynamic algorithms [4],

- It must require prior knowledge of global status of distributed system.

- It does not consider the current state or behaviour of a node while allocating the load.

- Its divide the traffic equivalently among all available server or VM.

Advantages: -

- High Stability.

- Utilize Less resources.

- More Predictability.

Disadvantages: -

- Not fault tolerant.

- Less reliable.

- It has less response time.

- Major influence on the overall system performance due to the randomness of load fluctuation.

Example: - Round Robin, Random load balancing algorithm, threshold load balancing algorithm.

Dynamic Load Balancing Algorithms: - In Dynamic load balancing:

- It considers the current state or behaviour of a node while allocating the load.

- It does not require prior knowledge of global status of distributed system.

- It is more comfortable for widely distributed systems like cloud computing.

- Its divides the traffic according to the capacity of all available servers or VMs.

- If any node fails, it will not break the system but it will only affect the system performance.

Advantages: -

- High Adaptive technique.

- Highly fault tolerant.

- High response time.

- It is highly reliable.

Disadvantages: -

- It is less stable.

- Utilize High resources.

- Less Predictability.

Example:-Active Monitoring, Throttle load balancing algorithm, Task scheduling algorithm, Enhance equally distributed load balancing algorithm.

\section{EXISTING LOAD BALANCING POLICY IN CLOUDANALYST}

There are various load balancing algorithms proposed in cloud computing research field but only three algorithms which exist in cloud analyst simulators has been focused

\subsection{Round Robin Load Balancing Policy}

Round robin is one of the straightforward and static scheduling technique that utilize the principle of time slices which divided time into multiple interval and each VM is given a particular time slice or time interval [8], [5].Round robin works on arbitrary selection of the VMs

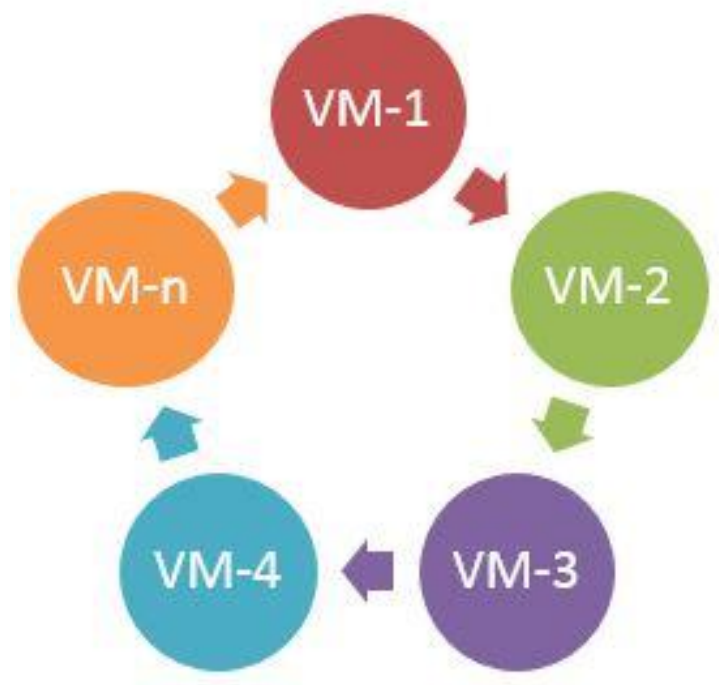

Fig 2: Round Robin Load Balancing

It assigns requests to a list of existing $\mathrm{VMs}$ on a rotational basis. The first request is assigned to a VM selected arbitrarily from the group and then the Data Centre controller allots the requests in a circular order. When the $\mathrm{VM}$ is assigned the request, the VM is progressed to the end of the list [7].

Drawback:-

- Without checking the capacity of sever, it's directly assign the request (like whether it is over loaded or not).

- It does not include the state of previous allocation of a VM to a request [8].

Existing Solutions: -

There are many improved load balancing algorithms proposed which help to understand problem of existing algorithm up to some extent in cloud environment. Some of them are:

- N. Pasha et al. [8] proposed an algorithm to addresses the problem with a round robin policy for" Round Robin Approach for VM Load Balancing Algorithm in Cloud Computing Environment".

- V. Darji et al. [2] proposed an algorithm "Dynamic Load Balancing For Cloud Computing Using Heuristic Data and Load on Server "In Dynamic Round Robin, strategy, weights assignments is based on continuous monitoring of the servers and is therefore constantly changing achieves optimum resource utilization.

\subsection{Active Monitoring Load Balancing (AMLB) Policy}

Active monitoring load balancing is dynamic in nature. It keeps information about each VM's and the number of request presently assigned to which $\mathrm{VM}$ when a request is allocate a new VM reaches. If there are more than one VM, the first recognized is selected and AMLB returns the VM id to the datacenter controller. The datacenter controller sends the request to the VM known by that VM id. The datacenter controller warns the AMLB to new allocation and request is sent to it [8] 


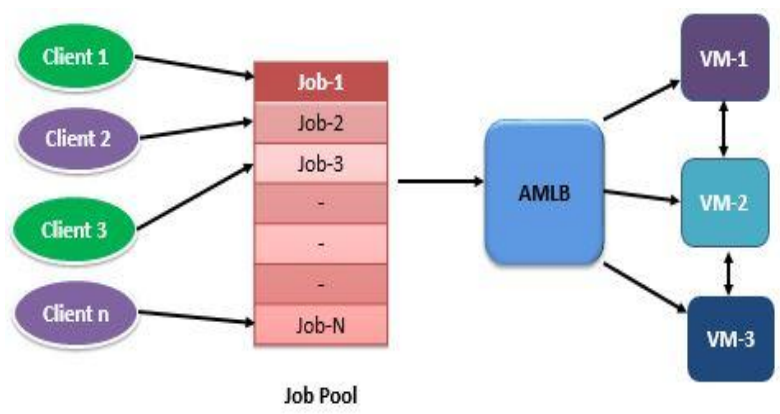

Fig 3: Active Monitoring Load Balancing [8]

\section{Drawback:-}

- AMLB always find least loaded VM for assign new incoming request but it will not check whether it's previously utilized or not (so some VM over utilized and some is still ideal)

Existing Solutions: -

- S. G.Damanal et al. [13] proposed an algorithm "Optimal Load Balancing in Cloud Computing by Efficient Utilization of Virtual Machines", this paper provide the solution to improve response time, efficient utilization of available resources. Proposed algorithm employs a method for selecting a VM.

\subsection{Throttled Load Balancing Policy}

Throttled algorithm is best in performance and in response time compare to existing two policies. It is also dynamic in behavior. It assigns all incoming job in efficient way to VM.

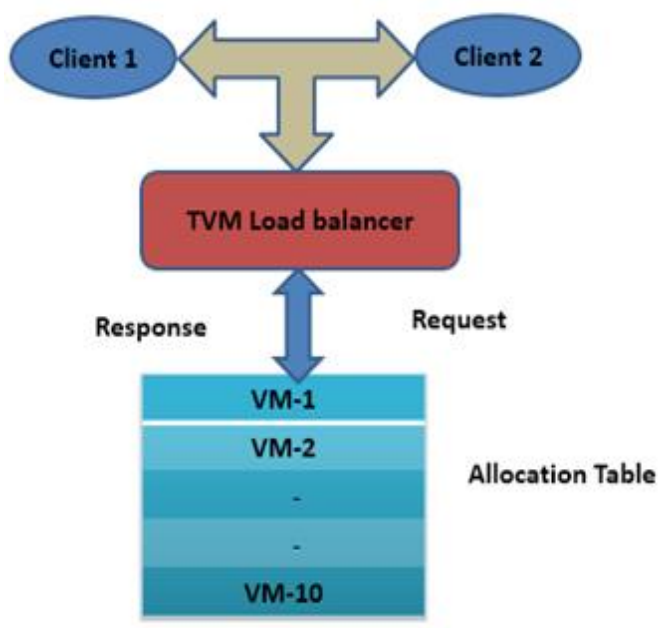

Fig 4: Throttled Load Balancing [8]

It discover the suitable virtual machine for assigning a specific job. The job manager is having a list of all virtual machines, using this indexed list, it allot the desire job to the suitable machine which access that load easily and complete the operations. If the job is well suited for a particular virtual machine in terms of size and availability of the virtual machine and that job assign to the suitable machine otherwise the job manager waits for the client request and put the job in queue for fast processing. This algorithm performs well as compared to round robin algorithm [3].

\section{Drawback:-}

- In throttled algorithm, where the index table is resolve from the first index every time when the data center queries load balancer for allocation of VM.
- It does not takes into account the advanced load balancing requirements such as processing times for each individual requests [14].

Existing Solutions: -

- S. G.Damanal et al. [7] proposed an algorithm "Load Balancing in Cloud Computing Using Modified Throttled Algorithm", this paper algorithm focuses mainly on how incoming jobs are assigned to the available virtual machines intelligently.

\section{CLOUDANALYST SIMULATOR}

The Cloud Analyst is assembled on top of CloudSim tool kit, which allows description of location of data centres, with information of geographic location of users generating traffic and application workloads, data centers, number of users and resources in each data center by extending CloudSim functionality [6].

Cloud Analyst fill the gap of present simulation tools for assessment of cloud environment and applications which enable developer to estimate requirement of large scale application in terms of geographic distribution of workload and computing server [6].

Main Features of the Cloud Analyst Simulator

- An easy to use tool with a high-level of visualization capability, which is even better than just a toolkit with necessary configurations parameter.

- Ability to enter and change configurations parameters quickly and easily with high flexibility.

- Simulation results is in the form of graph and table which help to analyzed smoothly, more efficiently and it may also help in quickly identify any problems with the performance and accuracy of the simulation logic [6].

- It allows to save the configuration as file (.sim) and allows to load configuration file for further simulation.

- Ease of extension means continuously to evolve existing load balancing policy with minimal effort with suitable framework to improve certain parameter.

\section{SIMULATED RESULTS COMPARATION OF EXISTING ALGORITHMS.}

Datacentre configuration which consists no. of VM, storage, processor speed, VM image size, bandwidth, memory, VM policy should be define under datacentre configuration tab. User grouping factor, request grouping factor, instruction length, services broker policy should be define under Advanced tab.

Table 1. Table captions should be placed above the table

\begin{tabular}{|l|c|}
\hline \multicolumn{1}{|c|}{ Parameter } & Value Used \\
\hline Request Per User Per Hour & 60 \\
\hline Data Size Per Request & 100 \\
\hline Number of region use & 6 \\
\hline Peak hour start(GMT) & 3 \\
\hline Peak hour end (GMT) & 9 \\
\hline DC 1- No Of VM & 5 \\
\hline VM Image Size & $10000 \mathrm{MB}$ \\
\hline VM Memory & $512 \mathrm{MB}$ \\
\hline VM Bandwidth & $1000 \mathrm{bps}$ \\
\hline DC 1- Storage Per Machine & $100000000 \mathrm{Mb}$ \\
\hline DC 1- Available BW Per Machine & 1000000 \\
\hline DC 1- No Of Processors Per Machine & 4 \\
\hline DC 1- Processor Speed & $10000 \mathrm{MIPS}$ \\
\hline
\end{tabular}




\begin{tabular}{|l|c|}
\hline DC 1- VM Policy & Time Shared \\
\hline User Grouping Factor & 10 \\
\hline Request Grouping Factor & 10 \\
\hline Executable Instruction Length & 100 \\
\hline Service broker policy & Closest DC \\
\hline
\end{tabular}

\begin{tabular}{|c|c|c|c|}
\hline & $\operatorname{Avg}(\mathrm{ms})$ & Min(ms) & $\operatorname{Max}(\mathrm{ms})$ \\
\hline Over All Response Time & 381.05 & 39.61 & 668.09 \\
\hline DC Processing Time & 0.90 & 0.01 & 10.23 \\
\hline \multicolumn{4}{|c|}{ Round Robin } \\
\hline & Avg(ms) & $\operatorname{Min}(\mathbf{m s})$ & $\operatorname{Max}(\mathrm{ms})$ \\
\hline Over All Response Time & 380.95 & 39.61 & 668.09 \\
\hline DC Processing Time & 0.80 & 0.01 & 6.63 \\
\hline
\end{tabular}

Equally Spread Current Execution Load

\begin{tabular}{|l|l|l|l|}
\hline & $\operatorname{Avg}(\mathrm{ms})$ & $\operatorname{Min}(\mathrm{ms})$ & $\operatorname{Max}(\mathrm{ms})$ \\
\hline Over All Response Time & 380.84 & 39.61 & 668.09 \\
\hline DC Processing Time & 0.68 & 0.01 & 3.87 \\
\hline
\end{tabular}

Throttled Load balancing

Fig 4: Results Comparison

\section{IMPLEMENTATION OF LOAD BALANCING POLICY IN CLOUDANALYST}

For implementing new approaches for load balancing policy, following classes have been changed to simulate above scenario.

Table 2. Classes need to change

\begin{tabular}{|l|c|c|}
\hline \multicolumn{1}{|c|}{ Package } & Class & Method \\
\hline cloudsim.ext.gui.screens & $\begin{array}{c}\text { ConfigureSimulati } \\
\text { onPanel }\end{array}$ & $\begin{array}{c}\text { createMain } \\
\text { Tab }\end{array}$ \\
\hline cloudsim.ext & Constants & - \\
\hline cloudsim.ext,datacenter & $\begin{array}{c}\text { datacentercontrol } \\
\text { ler }\end{array}$ & - \\
\hline cloudsim.ext.datacenter & $\begin{array}{c}\text { Add new class } \\
\text { Newloadbalancer }\end{array}$ & - \\
\hline $\begin{array}{l}\text { cloudsim.ext.datacenter } \\
\text { (for new policy) }\end{array}$ & Newloadbalancer & $\begin{array}{c}\text { getnextavail } \\
\text { ableVm }\end{array}$ \\
\hline
\end{tabular}

\section{CONCLUSION AND FUTURE WORK}

From above survey of load balancing and three existing policies of cloud analyst simulator, one can conclude that, designing of load balancing algorithms is challenging task in cloud computing. In this survey paper, what the load balancing exactly is and how its work according to their behavior is shown in this paper. To the best of our knowledge in load balancing concept, once simulator with three default load balancing policy were compared with different parameter such as response time and performance and also discussed their problem with existing solutions. Ultimately we know the important of load balancing in cloud computing which help in better resources utilization to gain high performance and better response time of system. In future you can edit your own modified policy by modifying all above mention classes according to your policy requirement, then compare different parameter with existing policies and analyze the behavior of new policy.

\section{REFERENCES}

[1] Landis, Cary, and Dan Blacharski. Cloud Computing Made Easy. Lulu Enterprices Incorporated, 2010.

[2] Darji, Vinay, Jayna Shah, and Rutvik Mehta. "Dynamic Load Balancing For Cloud Computing Using Heuristic Data and Load on Server." (IOSR-JCE) Volume 16, Issue 4, (Jul - Aug. 2014), PP 59-69.

[3] H. Mahalle, P. Kaveri, V. Chavan, "Load Balancing on Cloud Data Centers", international Journal of Advance Research in computer Science and Software Engineering, vol. 3, pages 11-15, Jan. 2013.

[4] Kaur, Jaswinder, and Supriya Kinger. "A Survey on Load Balancing Techniques in Cloud Computing." IJSR 2012 Pages-2662-2665.

[5] Shah, MR Manan D., MR Amit A. Kariyani, and MR Dipak L. Agrawal. "Allocation of Virtual Machines in Cloud Computing Using Load Balancing Algorithm." (IJCSITS), ISSN (2013): 2249-9555.

[6] B. Wickremasinghe and R. N. Calheiros, "CloudAnalyst: a cloudsim-based visual modeller for analysing cloud computing environments and applications," 24th International Conference on Advanced Information Networking and Application, pp.446-452, 2010.

[7] Domanal, S. G., \& Reddy, G. R. M. (2013, October). Load Balancing in Cloud Computing using Modified Throttled Algorithm. In Cloud Computing in Emerging Markets, 2013 IEEE International Conference on (pp. 15). IEEE.

[8] Nusrat Pasha, Dr. Amit Agrawal, Dr. Ravi Rastogi, "Round Robin Approach for VM Load Balancing Algorithm in Cloud Computing Environment", IJARCSSE, Volume 4, pages 34-39 Issue 5, May 2014.

[9] Qi Zhang, Lu Cheng, Raouf Boutaba. "Cloud computing: state-of-the-art and research challenges", J Internet ServAppl (2010) 1: 7-18, Springer

[10] Jianzhe Tai, JueminZhang,JunLi,Waleed Meleis and NingfangMi “A R A: Adaptive Resource Allocation for Cloud Computing Environments under Bursty Workloads" 978-1-4673-0012-4/11 @2011 IEEE.

[11] Wei Zhao, Yong Peng, Feng Xie, Zhonghua Dai (2012). "Modeling and Simulation of Cloud Computing: A Review", IEEE Asia Pacific Cloud Computing Congress, pages 20-24, 2012.

[12] NIST Definition of Cloud Computing v15, csrc.nist.gov/groups/SNS/cloud-computing/cloud-defv15.doc

[13] Domanal, Shridhar G., and G. Ram Mohana Reddy."Optimal load balancing in cloud computing by efficient utilization of virtual machines." COMSNETS 2014. 2014, Page(s): 1- 4

[14] Bhatia, J., Patel, T., Trivedi, H., \& Majmudar, V. (2012, December). HTV Dynamic Load Balancing Algorithm for Virtual Machine Instances in Cloud. InCloud and Services Computing (ISCOS), 2012 International Symposium on (pp. 15-20). IEEE.

[15] http://en.wikipedia.org/wiki/Load_balancing_\%28compu ting $\% 29$

[16] Kaur, Rajwinder, and Pawan Luthra. "Load Balancing in Cloud Computing."Proceedings of International Conference on Recent Trends in Information, Telecommunication and Computing, ITC. 2012. 